\title{
Chemical and Textural Properties in Commercial Fermented Soybean Curds of Sufu
}

\author{
Joo-Shin Kim**, Ying Lu, and Hau Yin Chung ${ }^{\dagger}$ \\ School of Life Sciences, Food and Nutritional Sciences Programme, The Chinese University \\ of Hong Kong, Shatin, N. T., Hong Kong
}

\begin{abstract}
A survey aiming to find out the chemical and textural properties of commercial fermented soy bean curd called sufu was conducted. Sixteen brands of plain sufu produced in the Northem or the Southem part of China were collected and examined for their crude protein, crude fat, texture profiles, free amino acids, and free fatty acid contents. Twenty-one free amino acids were extracted and derivatized using a commercial kit followed by separation and analyzed by the gas chromatography-mass spectrometry (GC-MS). Similary, ten free fatty acids were extracted using alumina, eluted, separated and analyzed. The content ranges of crude fat and protein were $22 \sim 36 \%$ and $31 \sim 38 \%$, respectively. In texture profile analysis, ranges of the texture parameters were $131 \sim 493 \mathrm{~g}$ (hardness), $0.4 \sim 0.5$ (cohesiveness), -137 to -50 gs (adhesiveness), $0.6 \sim 1$ (springiness), $47 \sim 220 \mathrm{~g}$ (gumminess) and $32 \sim 177$ $\mathrm{g}$ (chewiness). Twenty-one different free amino acids, especially alanine, glycine, $\alpha$-aminobutyric acid, valine, leucine, allo-isoleucine, aspartic acid, glutamic acid and lysine in large amount, as well as ten fatty acids in total, notably linoleic acid (9-octadecanoic acid), oleic acid $(9,12$-octadecadienoic acid), linolenic acid $(9,12,15$-octadecadienoic acid), hexadecanoic acid and octadecanoic acid were found. This information provides important quality reference ranges for product developers and manufacturers to optimize and produce the plain sufu.
\end{abstract}

Key words: fermented soybean curd, sufu, free amino acid, free fatty acid, texture

\section{INTRODUCTION}

Fermented soybean curd or sufu, originated in China, is a soft creamy cheese-type product made from soybean curd by the fermentation of mould $(1,2)$. This product with its nutrition and characteristic flavor has been widely consumed by the natives as an appetizer or seasoning for many centuries. The maturation period for sufu production usually takes three to six months for component hydrolysis resulting in textural changes and flavour intensity increases. During that stage, hydrolysis converts macromolecules to small molecules such as peptides, amino acids, amines, ammonia, triglyceride, fatty acids making it a good source of digestive amino acids and fatty acids in the Chinese diet from the nutritional point of view. Furthermore, both protein and fat fractions have effects on its texture, and on development of the sufu flavor (3).

There are only a handful of publications describing the texture profile, amino acid profile and fatty acid profile in sufu, because during the fermentation, many changes take place. It is rather complicated to monitor individual amino acid, fatty acid and many other parameters at the same time. Even as such, the knowledge of chemical and biological properties of sufu is important to understand the fundamentals of food fermentation and further investigation in the sufu system. Therefore, the primary objective in this study was to determine the various qualities in the commercial plain sufus. The investigation covered from the basic crude protein, crude fat and texture profile to an in-depth monitoring of individual free amino acid and free fatty acids hoping the data will be eventually served as references for research-purposed laboratory-scale sufu production, control and improvement.

\section{MATERIALS AND METHODS}

\section{Sufu sampling}

Sixteen brands $(\mathrm{D} \sim \mathrm{F}, \mathrm{H} \sim \mathrm{T})$ of white commercial sufu were purchased from both the Northern and Southern China. Among them, brand $\mathrm{F}$ and $\mathrm{H}$ were produced in Beijing; brand $\mathrm{E}$ was manufactured in Hunan province; brand $\mathrm{K}, \mathrm{R}$ and $\mathrm{S}$ were produced in Hong Kong; brand $\mathrm{D}, \mathrm{J}, \mathrm{L}, \mathrm{M}, \mathrm{O}, \mathrm{P}, \mathrm{Q}$ and $\mathrm{T}$ were manufactured in several cities of Guangdong province; brand I and $\mathrm{N}$ were produced in Taiwan. Brands from the South were a few more because plain sufu is mainly consumed in Southern

\footnotetext{
*This author is also affiliated to the Kwangil Synthesis Plant Co. Ltd.

${ }^{\dagger}$ Corresponding author. E-mail: anthonychung@cuhk.edu.hk

Phone: +852-2609-6149, Fax: +852-2603-5745
} 
China, and manufacturers centralize in the South. Samples were all well-chosen randomly and all of them represented sufu of different origins.

\section{Crude protein analysis}

Crude protein of sufu samples was determined according to Kjeldahl method AOAC No. 987.02 (4) using Kjeltec Systems with Digester 2006 and Distiller 1002 (FOSS Tecator AB, Hoganas, Sweden). Protein content was calculated by multiplying the nitrogen content by a factor of 6.25 (5) and expressed as the concentration of protein in the freeze-dried sufu samples (protein $\%$ dry mass weight).

\section{Crude fat analysis}

Crude fat was measured by a Soxtec System HT 1043 Extraction Unit (FOSS Tecator $\mathrm{AB}$ ) according to AOAC No. 39.1.08 (6).

\section{Free amino acid analysis}

A "EZ: Faast GC-MS for free amino acid analysis" kit purchased from Phenomenex (EZ: Faast TM KGO7166, Phenomenex ${ }^{\circledR}$, Torrance, CA, USA) which contained all amino acid standards and reagents used in the SPE (Solid Phase Extraction) and derivatization steps was used to analyze the free amino acids. Sample pretreatment was required before using the kit. Briefly, two grams of fresh sufu was manually grinded and sonicated for $30 \mathrm{~min}$ with $10 \mathrm{~mL}$ of distilled water in a capped plastic bottle in order to dissolve the free amino acid in water (7). The sample was centrifuged at $16000 \times g$, $4^{\circ} \mathrm{C}$ for $15 \mathrm{~min}$ after $5 \mathrm{~mL}$ of water was added. Supernatant was filtered through a $0.45-\mu \mathrm{m}$ pore size HA membrane filter (Millipore DURAPORE® Cat No.: HVLP02500, $0.45 \mu \mathrm{m} \mathrm{HV}$, Millipore Ireland, Cork, Ireland), collected and stored at $-70^{\circ} \mathrm{C}$ before further analysis (8).

Free amino acid gas chromatography-mass spectrometry (GC-MS) conditions

The derivatized free amino acids present in the standards and samples were separated and identified by the GC-MS. A $10 \mathrm{~m} \times 0.25 \mathrm{~mm}$ Zebron TM ZB-AAA gas chromatography column comes with the EZ: Faast TM was installed in the GC. The helium carrier gas was set at a constant flow of $1.1 \mathrm{~mL} / \mathrm{min}$. Temperature of GC oven increased from $110^{\circ} \mathrm{C}$ to $320^{\circ} \mathrm{C}$ at the rate of $30^{\circ} \mathrm{C} /$ min. Injection port temperature was $250^{\circ} \mathrm{C}$ with split ratio of $15: 1$. MS temperatures were set to $240^{\circ} \mathrm{C}$ for ion source, $180^{\circ} \mathrm{C}$ for the quadrupole and $130^{\circ} \mathrm{C}$ for MSD auxiliary unit. The scan range was $45 \sim 450 \mathrm{~m} / \mathrm{z}$. Sample injection volume was $2 \mu \mathrm{L}$ each time.

\section{Free fatty acid determination}

The method was adapted from Deeth et al. (9). Briefly, one gram of fresh sufu was grinded and mixed with five $\mathrm{mL}$ of diethyl ether containing $100 \mu \mathrm{g}$ of each C5:0 and $\mathrm{C} 17: 0,0.1 \mathrm{~mL}$ of $4 \mathrm{~N}-\mathrm{H}_{2} \mathrm{SO}_{4}$ and $2.5 \mathrm{~g}$ of granular anhydrous sodium sulfate. After stood for $1 \mathrm{hr}, 5 \mathrm{~mL}$ of hexane was added. The mixture was centrifuged at 2000 $\times g$ for $5 \mathrm{~min}$ at $4^{\circ} \mathrm{C}$ and the supernatant passed through twice a Pasteur pipette containing $1 \mathrm{~g}$ of neutral alumina oxide (ALOX 90N, 71-077-2.0 NA Chemicals, Art.) (9). Two $\mathrm{mL}$ of diisopropyl ether containing $6 \%$ of formic acid was added to the alumina followed by centrifugation at $2000 \times \mathrm{g}$ for 5 minutes at room temperature to free the fatty acid (9). The extracts were injected into the GC-MS.

\section{Free fatty acid GC-MS conditions}

The free fatty acids present in standards and extracted from sufu samples were analyzed using Agilent GC-MS system as before. The flow rate of helium was set at $1.5 \mathrm{~mL} / \mathrm{min}$ during the run and at $1.0 \mathrm{~mL}$ for the post run. A 30-m HP-5MS (0.25 mm i.d., $0.25 \mu \mathrm{m}$ df, Agilent Technologists, Santa Clara, CA, USA) column was installed in the GC. Oven temperature program was: $110^{\circ} \mathrm{C}$ for $3 \mathrm{~min}$, and raised to $310^{\circ} \mathrm{C}$ at the ramp rate of $35^{\circ} \mathrm{C}$ $/ \mathrm{min}$ for $5 \mathrm{~min}$. Temperature of the post run was set to $310^{\circ} \mathrm{C}$ and held for $8 \mathrm{~min}$. Injection port temperature was $280^{\circ} \mathrm{C}$ in splitless mode. For the MS, the ion source was set at $230^{\circ} \mathrm{C}$, quadrupole was at $150^{\circ} \mathrm{C}$ and the MSD auxiliary unit was held at $310^{\circ} \mathrm{C}$. The scan range was set to $70 \sim 500 \mathrm{~m} / \mathrm{z}(3.5 \mathrm{scans} / \mathrm{s})$. Sample injection volume was $2 \mu \mathrm{L}$.

\section{Compound identification and quantification}

The presence of each amino acid and fatty acid were confirmed by their retention time and mass spectrum with that of the authentic standard. For quantification, 3-point calibration curve was derived for each authentic standard.

\section{Texture profile analysis (TPA)}

In this investigation, one sufu cube randomly picked from the aging sample jars was placed on the base-plate of TA-XTi Texture Analyzer (Stable Micro Systems, Surrey, England). Each sample was compressed by an alumina cylinder probe to $30 \%$ of its original height. The parameters of the instrument were set as follows: pretest speed at $1.0 \mathrm{~mm} / \mathrm{sec}$, test speed at $1.0 \mathrm{~mm} / \mathrm{sec}$, post- test speed at $1.0 \mathrm{~mm} / \mathrm{sec}, 5 \mathrm{sec}$ of delay between the two bites, trigger force at $5.0 \mathrm{~g}$ and data acquisition rate at 200 pps. The data was analyzed using Texture Expert Version 1.22 Software (Stable Micro Systems) to measure hardness, cohesiveness, adhesiveness, springiness and gumminess as described by Bourne (10). All experiments were performed in triplicate. 


\section{Statistical analysis}

Concentrations of compounds from the three samples were analyzed by one-way analysis of variance (ANOVA) and compared by the Tukey test at $p<0.05$ level of significance using SPSS 10.0 (SPSS Inc., Chicago, IL, USA). Concentrations of compounds from both fresh and deep-fat fried samples were analyzed by student $t$-test at $\mathrm{p}=0.05$ level of significance.

\section{RESULTS AND DISCUSSION}

Crude protein and fat profiles of the sixteen different commercial brands were compared by one-way ANOVA and found out that at significant level of $p<0.05$, there was evidence that different brands of sufu were inconsistent. The mean protein and fat content $(\%$, dry wt basis) in the sufus were $35 \pm 1$ and $27 \pm 1$, respectively. Products from southern China had higher protein content (S. China $>$ N. China $>$ Taiwan $>$ Hong Kong: 36\%> 35\% $>34 \%>33 \%$ ). For fat content, products from Taiwan (35\%) had the highest quantity followed by Hong Kong (28\%), S. China (26\%) and N. China (23\%) (Table 1).

Texture profiles of the sixteen different commercial brands and their one-way ANOVA results (Table 1) showed that hardness, cohesiveness, gumminess and chewiness of the sixteen commercial brands were statistically the same from one brand to another, respectively at significant level of 0.05 . For adhesiveness, only brand $\mathrm{L}$ and brand $\mathrm{T}$ were different from brand $\mathrm{Q}$, while for springiness, only brand I was not the same as brand $\mathrm{N}$. Overall the texture profiles were quite similar for the sixteen brands of sufu. As shown in the texture profile analysis of Table 1 , the mean values for the hardness, cohesiveness, adhesiveness, springiness, gumminess and chewiness were $254 \pm 47 \mathrm{~g}, 0.4 \pm 0.0,-75 \pm 12 \mathrm{~g}, 0.7 \pm$ $0.0,85 \pm 20 \mathrm{~g}$ and $59 \pm 17 \mathrm{~g}$, respectively. Among the different regions, products from both N. China and Taiwan had the hardest of over $300 \mathrm{~g}$. Products from Hong Kong $(182 \mathrm{~g})$ were the softest compared with that from S. China $(236 \mathrm{~g})$. Cohesiveness was similar among products from N. and S. China and Hong Kong (0.4). Products of Taiwan also had cohesiveness of 0.4. For adhesiveness, products from Hong Kong and Taiwan were similar $(-82 \mathrm{~g})$ and were weaker than that from N. China $(-74 \mathrm{~g})$ and S. China $(-71 \mathrm{~g})$. For springiness, product of Taiwan has the highest value of 0.8 while the rest of the products were slightly lower. Highest and lowest gumminess were found in products from N. China $(128 \mathrm{~g})$ and Hong Kong $(62 \mathrm{~g})$. Texture profiles were relatively stable among the different commercial sufus. It might be because the texture properties were basically affected by the composition of protein, fat and water etc. (11). After the hydrolysis of protein and fat diminished, the major chemical activities might have taken place for the small molecules, such as the catabolism of free amino acids and free fatty acids, namely the deamination, transamination and decarboxylation of amino acids $(12,13)$, the esterification and microbial $\beta$-oxidation of fatty acids (14). Although the amounts of small molecules determined might be different resulted from the above activities, the amount ratio (protein-fat-water-etc.) might be quite stable in the maturated sufu after

Table 1. Crude protein, fat and texture profiles of different sufu brands

\begin{tabular}{|c|c|c|c|c|c|c|c|c|}
\hline & \multirow{2}{*}{$\begin{array}{c}\text { Crude protein } \\
(\% \text { dry } \\
\text { weight } \pm \text { SE })\end{array}$} & \multirow{2}{*}{$\begin{array}{c}\text { Crude fat } \\
(\% \text { dry } \\
\text { weight } \pm \text { SE })\end{array}$} & \multicolumn{6}{|c|}{ Texture profile $($ mean $\pm \mathrm{SE})$} \\
\hline & & & Hardness/g & Cohesiveness & Adhesiveness/gs & Springiness & Gumminess/g & Chewiness $/ \mathrm{g}$ \\
\hline $\mathrm{D}$ & $38 \pm 1^{\text {ac }}$ & $25 \pm 2^{\text {acdf }}$ & $357 \pm 7$ & $0.4 \pm 0.0$ & $-83 \pm 9$ & $0.7 \pm 0.0$ & $125 \pm 9$ & $91 \pm 7$ \\
\hline $\mathrm{E}$ & $34 \pm 1$ & $22 \pm 1^{\mathrm{cf}}$ & $493 \pm 243$ & $0.5 \pm 0.1$ & $-67 \pm 8$ & $0.7 \pm 0.1$ & $220 \pm 132$ & $177 \pm 130$ \\
\hline $\mathrm{F}$ & $38 \pm 1^{\mathrm{c}}$ & $24 \pm 2^{\text {adf }}$ & $306 \pm 47$ & $0.4 \pm 0.0$ & $-72 \pm 9$ & $0.7 \pm 0.1$ & $95 \pm 20$ & $66 \pm 23$ \\
\hline $\mathrm{H}$ & $33 \pm 1^{\text {be }}$ & $24 \pm 1.2^{\mathrm{adf}}$ & $218 \pm 13$ & $0.4 \pm 0.0$ & $-83 \pm 7$ & $0.7 \pm 0.1$ & $69 \pm 6$ & $47 \pm 8$ \\
\hline I & $34 \pm 1$ & $33 \pm 1^{b}$ & $387 \pm 130$ & $0.4 \pm 0.1$ & $-105 \pm 5$ & $0.6 \pm 0.1^{\mathrm{a}}$ & $115 \pm 35$ & $64 \pm 15$ \\
\hline $\mathrm{J}$ & $35 \pm 1$ & $28 \pm 1^{\mathrm{dg}}$ & $131 \pm 9$ & $0.4 \pm 0.0$ & $-55 \pm 5$ & $0.7 \pm 0.0$ & $47 \pm 3$ & $34 \pm 3$ \\
\hline $\mathrm{K}$ & $35 \pm 0^{\text {acde }}$ & $26 \pm 0^{\text {adf }}$ & $206 \pm 10$ & $0.4 \pm 0.0$ & $-84 \pm 12$ & $0.6 \pm 0.0$ & $72 \pm 8$ & $44 \pm 6$ \\
\hline $\mathrm{L}$ & $37 \pm 0^{\text {acd }}$ & $26 \pm 1^{\text {defg }}$ & $300 \pm 33$ & $0.4 \pm 0.0$ & $-50 \pm 12^{\mathrm{a}}$ & $0.6 \pm 0.1$ & $87 \pm 10$ & $55 \pm 11$ \\
\hline $\mathrm{M}$ & $34 \pm 1$ & $25 \pm 0^{\text {adf }}$ & $204 \pm 22$ & $0.5 \pm 0.0$ & $-54 \pm 3$ & $0.7 \pm 0.0$ & $73 \pm 2$ & $49 \pm 2$ \\
\hline $\mathrm{N}$ & $33 \pm 1^{\text {bde }}$ & $36 \pm 0^{\mathrm{b}}$ & $223 \pm 10$ & $0.4 \pm 0.0$ & $-58 \pm 7$ & $1 \pm 0^{\mathrm{b}}$ & $61 \pm 7$ & $61 \pm 9$ \\
\hline $\mathrm{O}$ & $35 \pm 1^{\text {acde }}$ & $28 \pm 2^{\text {de }}$ & $158 \pm 33$ & $0.4 \pm 0.0$ & $-61 \pm 11$ & $0.6 \pm 0.1$ & $50 \pm 12$ & $32 \pm 9$ \\
\hline $\mathrm{P}$ & $37 \pm 1^{\text {acd }}$ & $27 \pm 1^{\text {defg }}$ & $256 \pm 66$ & $0.4 \pm 0.0$ & $-75 \pm 26$ & $0.6 \pm 0.0$ & $81 \pm 19$ & $51 \pm 14$ \\
\hline Q & $36 \pm 1^{\text {acde }}$ & $23 \pm 1^{\text {adf }}$ & $283 \pm 39$ & $0.4 \pm 0.0$ & $-137 \pm 43^{b}$ & $0.7 \pm 0.1$ & $84 \pm 13$ & $57 \pm 8$ \\
\hline $\mathrm{R}$ & $31 \pm 1^{\mathrm{b}}$ & $32 \pm 1^{\text {beg }}$ & $177 \pm 23$ & $0.4 \pm 0.0$ & $-67 \pm 4$ & $0.6 \pm 0.0$ & $60 \pm 9$ & $38 \pm 7$ \\
\hline $\mathrm{S}$ & $34 \pm 0^{\mathrm{ab}}$ & $27 \pm 1^{\text {defg }}$ & $163 \pm 27$ & $0.4 \pm 0.0$ & $-94 \pm 18$ & $0.7 \pm 0.0$ & $53 \pm 9$ & $37 \pm 6$ \\
\hline $\mathrm{T}$ & $36 \pm 0^{\text {acde }}$ & $25 \pm 1^{\text {adg }}$ & $199 \pm 45$ & $0.4 \pm 0.0$ & $-52 \pm 8^{a}$ & $0.7 \pm 0.0$ & $68 \pm 19$ & $46 \pm 12$ \\
\hline Mean & 35 & 27 & 254 & 0.4 & -75 & 0.7 & 85 & 59 \\
\hline
\end{tabular}

Data are presented as mean \pm SE for 3 replicates. The values with different lowercase letters differ with each other at the significant level of $\mathrm{p}<0.05$. The mean values with no lowercase letter mean that this property in this brand is nearly the same as that in all other brands. 
complete hydrolysis. If the initial content of protein in soybean was close in different brands, under similar production conditions, the final amount ratio of proteinfat-water-etc. should be quite consistent, making the texture profile uniform. It is also implied that hydrolysis took only a short period in aging. After that, hydrolysis was nearly done and other chemical activities play more important role. Aroma compounds such as flavor esters transformed from fatty acids were mainly formed in this post-hydrolysis period.

The corresponding mass charge fragment ions of the derivatized common free amino acids in standards, used to confirm the identification of the amino acid peaks and quantify their amounts, were listed in Table 2 . Thirty-three standard amino acids in total, including the internal standard, were positively separated in $8 \mathrm{~min}$. The results of every single free amino acid in all sixteen different brands of commercial samples were listed in Table 3 . Twenty-one free amino acids in total were positively identified and quantified. The total free amino acid contents ranged from $100 \mathrm{nmol} / \mathrm{g}$ in brand I to $194 \mathrm{nmol} / \mathrm{g}$ in brand $\mathrm{N}$ among 16 brands. Among the sixteen brands,
ALA, GLY, ABA, VAL, LEU, aILE, ASP, GLU and LYS were higher in amount than others, while the amount of ALA was especially the highest. The mean total amount of free amino acids was $139 \mathrm{nmole} / \mathrm{g}$ fresh sample. Among the regions, products of Taiwan had the highest free amino acid contents (147 nmole/g fresh sample) and were followed by S. China (142), Hong Kong (141) and N. China (123) (Tables 2 and 3).

The corresponding mass fragment ions of the derivatized common free fatty acids in standards were listed in Table 4. Thirty fatty acid standards in total (including the internal standard) were positively separated in 10 min. Results of free fatty acids in all sixteen different brands of commercial samples were listed in Table 5 . Ten free fatty acids in total positively identified and quantified in all sixteen different commercial sufu samples. The total free fatty acid contents ranged from $299 \mathrm{mg} / 100 \mathrm{~g}$ in brand $\mathrm{E}$ to $1203 \mathrm{mg} / 100 \mathrm{~g}$ in brand L. The mean total amount of free fatty acids was 747 $\mathrm{mg} / 100 \mathrm{~g}$ of fresh sample. Among the sixteen brands, fatty acids $\mathrm{C}_{18: 2(\mathrm{n}-6)}, \mathrm{C}_{18: 1(\mathrm{n}-9)}, \mathrm{C}_{16: 0}, \mathrm{C}_{18: 0}$ and $\mathrm{C}_{18: 3}$ were higher in amount than other fatty acids, while that of

Table 2. Retention times, elution order, names, abbreviations and ion fragments of the 33 amino acid standards

\begin{tabular}{|c|c|c|c|c|}
\hline Rt (min) & Elution order & Chemical name & Abbreviation & Mass fragment ions \\
\hline 1.19 & 1 & Alanine & ALA & 130,88 \\
\hline 1.26 & 2 & Sarcosine & SAR & 130,217 \\
\hline 1.30 & 3 & Glycine & GLY & 116,207 \\
\hline 1.40 & 4 & $\alpha$-Aminobutyric acid & $\mathrm{ABA}$ & 144,102 \\
\hline 1.50 & 5 & Valine & VAL & 158,116 \\
\hline 1.57 & 6 & $\beta$-Aminoisobutyric acid & $\beta-\mathrm{AiB}$ & 116,158 \\
\hline 1.63 & 7 & Norvaline (internal standard) & NORV (I.S.) & 158,72 \\
\hline 1.71 & 8 & Leucine & LEU & 172,86 \\
\hline 1.74 & 9 & allo-Isoleucine & aILE & 172,130 \\
\hline 1.81 & 10 & Isoleucine & ILE & $172,130,69$ \\
\hline 1.98 & 11 & Threonine & THR & 101,160 \\
\hline 2.03 & 12 & Serine & SER & 203,146 \\
\hline 2.09 & 13 & Proline & PRO & 156,243 \\
\hline 2.19 & 14 & Asparagine & ASN & 69,155 \\
\hline 2.55 & 15 & Thioproline & TPR & $88,174,147$ \\
\hline 2.76 & 16 & Aspartic acid & ASP & 216,130 \\
\hline 2.78 & 17 & Methionine & MET & 203,277 \\
\hline 2.93 & 18 & 4-Hydroxyproline & $4 \mathrm{HYP}$ & 172,86 \\
\hline 3.12 & 19 & Glutamic acid & GLU & $84,230,170$ \\
\hline 3.14 & 20 & Phenylalanine & PHE & $148,206,190$ \\
\hline 3.43 & 21 & $\alpha$-Aminoadipic acid & AAA & 98,244 \\
\hline 3.70 & 22 & $\alpha$-Aminopimelic acid & APA & $198,258,286$ \\
\hline 3.77 & 23 & Glutamine & GLN & 84,187 \\
\hline 4.17 & 24 & Ornithine & ORN & 156,70 \\
\hline 4.21 & 25 & Glycyl-proline & GPR & 70,300 \\
\hline 4.44 & 26 & Lysine & LYS & 170,128 \\
\hline 4.63 & 27 & Histidine & HIS & $81,282,168$ \\
\hline 4.80 & 28 & Hydroxylysine & HLY & 129,169 \\
\hline 4.91 & 29 & Tyrosine & TYR & 107,206 \\
\hline 5.14 & 30 & Proline-hydroxyproline & PHP & 156,184 \\
\hline 5.20 & 31 & Tryptophan & TRP & 130 \\
\hline 5.70 & 32 & Cystathionine & $\mathrm{CTH}$ & 203,272 \\
\hline 5.92 & 33 & Cystine & $\mathrm{C}-\mathrm{C}$ & $174,248,216$ \\
\hline
\end{tabular}


Table 3. Free amino acid contents of sufu brands

\begin{tabular}{|c|c|c|c|c|c|c|c|c|c|c|c|}
\hline \multirow{2}{*}{ Brand } & \multicolumn{11}{|c|}{$\begin{array}{l}\text { Free amino acid concentration ( } \mathrm{nmol} / \mathrm{g} \text { fresh weight of sufu) } \\
\text { (mean } \pm \mathrm{SE} \text { for } 3 \text { replicates })\end{array}$} \\
\hline & ALA & GLY & $\mathrm{ABA}$ & VAL & LEU & aILE & PRO & ASN & ASP & MET & GLU \\
\hline D & $29 \pm 1$ & $13 \pm 2$ & $4.4 \pm 0.7^{\mathrm{ad}}$ & $14 \pm 1$ & $22 \pm 0$ & $13 \pm 0^{\mathrm{acd}}$ & $1 \pm 0^{\mathrm{a}}$ & $0.1 \pm 0.0^{\mathrm{ac}}$ & $3.9 \pm 0.6^{\mathrm{a}}$ & $2.3 \pm 0.2$ & $5.9 \pm 0.0^{\mathrm{ae}}$ \\
\hline $\mathrm{E}$ & $26 \pm 1$ & $13 \pm 0$ & $8.8 \pm 0.2^{\text {be }}$ & $13 \pm 0$ & $20 \pm 1$ & $11 \pm 0$ & $2.1 \pm 0.8^{\mathrm{a}}$ & $0.5 \pm 0.2^{\mathrm{c}}$ & $1.8 \pm 0.2^{\mathrm{a}}$ & $1.6 \pm 0.5$ & $3.8 \pm 0.9^{\mathrm{ae}}$ \\
\hline $\mathrm{F}$ & $12 \pm 0^{\mathrm{ad}}$ & $18 \pm 1^{\mathrm{a}}$ & $10 \pm 1^{\text {be }}$ & $12 \pm 2$ & $18 \pm 4$ & $11 \pm 2$ & $2.5 \pm 1.2^{\mathrm{ac}}$ & $0.03 \pm 0.00^{\mathrm{a}}$ & $6.4 \pm 0.2^{\mathrm{a}}$ & $1.9 \pm 0.4$ & $2.7 \pm 0.0^{\mathrm{a}}$ \\
\hline $\mathrm{H}$ & $36 \pm 2^{b c}$ & $17 \pm 1^{\mathrm{a}}$ & $10 \pm 0^{\text {be }}$ & $15 \pm 1^{\mathrm{ac}}$ & $21 \pm 0$ & $12 \pm 0^{\mathrm{acd}}$ & $1.5 \pm 0.5^{\mathrm{a}}$ & $0.02 \pm 0.00^{\mathrm{a}}$ & $3.5 \pm 0.3^{\mathrm{a}}$ & $2.4 \pm 0.0$ & $5.9 \pm 0.2^{\mathrm{ae}}$ \\
\hline I & $12 \pm 1^{\mathrm{ad}}$ & $10 \pm 1$ & $0.4 \pm 0.0^{\mathrm{ac}}$ & $7 \pm 1^{b}$ & $16 \pm 1^{\mathrm{ac}}$ & $6 \pm 1^{\text {bd }}$ & $5.9 \pm 0.0^{\mathrm{b}}$ & $0.1 \pm 0.0^{\mathrm{ac}}$ & $9.4 \pm 1.9$ & $1.5 \pm 0.1$ & $15 \pm 2^{\mathrm{b}}$ \\
\hline $\mathrm{J}$ & $20 \pm 7^{\text {ace }}$ & $12 \pm 2$ & $7.1 \pm 1.2^{\mathrm{de}}$ & $10 \pm 2^{\mathrm{bc}}$ & $14 \pm 2^{\mathrm{a}}$ & $9 \pm 1^{b c d}$ & $5.8 \pm 0.9^{b}$ & $0.1 \pm 0.0^{\mathrm{a}}$ & $9 \pm 2$ & $1.3 \pm 0.3^{\mathrm{a}}$ & $1.9 \pm 0.1^{\mathrm{a}}$ \\
\hline $\mathrm{K}$ & $32 \pm 3^{b c}$ & $15 \pm 2$ & $10 \pm 1^{\text {be }}$ & $14 \pm 2$ & $21 \pm 2$ & $12 \pm 1^{\text {acd }}$ & $0.8 \pm 0.3^{\mathrm{a}}$ & $0.1 \pm 0.0^{\mathrm{ac}}$ & $6 \pm 2^{a}$ & $2.2 \pm 0.2$ & $4.4 \pm 1.0^{\mathrm{ae}}$ \\
\hline $\mathrm{L}$ & $32 \pm 1^{b c}$ & $13 \pm 0$ & $10 \pm 0^{\text {be }}$ & $14 \pm 1$ & $20 \pm 1$ & $13 \pm 1^{\text {acd }}$ & $0.9 \pm 0.3^{\mathrm{a}}$ & $0.2 \pm 0.0^{\mathrm{ac}}$ & $7 \pm 2^{\mathrm{a}}$ & $2 \pm 0$ & $5.6 \pm 1.3^{\mathrm{ae}}$ \\
\hline $\mathrm{M}$ & $14 \pm 1^{\mathrm{cd}}$ & $13 \pm 2$ & $2 \pm 0^{\mathrm{ac}}$ & $11 \pm 1$ & $16 \pm 1^{\mathrm{ac}}$ & $10 \pm 1^{\mathrm{d}}$ & $5.4 \pm 0.6^{\mathrm{bc}}$ & $0.16 \pm 0.05^{\mathrm{ac}}$ & $17 \pm 4^{\mathrm{b}}$ & $1.5 \pm 0.1$ & $2.1 \pm 0.0^{\mathrm{a}}$ \\
\hline $\mathrm{N}$ & $16 \pm 1^{\mathrm{cd}}$ & $18 \pm 2^{\mathrm{a}}$ & $0.2 \pm 0.0^{\mathrm{c}}$ & $13 \pm 2$ & $19 \pm 2$ & $12 \pm 1$ & $1.2 \pm 0.0^{\mathrm{a}}$ & $0.1 \pm 0.0^{\mathrm{ac}}$ & $17 \pm 3^{\mathrm{b}}$ & $1.8 \pm 0.0$ & $75 \pm 3^{c}$ \\
\hline $\mathrm{O}$ & $40 \pm 2^{b}$ & $15 \pm 1$ & $12 \pm 1^{b}$ & $18 \pm 2^{\mathrm{a}}$ & $27 \pm 2^{b c}$ & $16 \pm 2^{\mathrm{a}}$ & $0.8 \pm 0.2^{\mathrm{a}}$ & $0.2 \pm 0.0^{\mathrm{ac}}$ & $7 \pm 1$ & $2.8 \pm 0.2^{\mathrm{b}}$ & $6.3 \pm 0.4^{\mathrm{ae}}$ \\
\hline $\mathrm{P}$ & $34 \pm 7^{\text {be }}$ & $15 \pm 3$ & $10 \pm 2^{\text {be }}$ & $16 \pm 3^{\mathrm{ac}}$ & $26 \pm 3^{c}$ & $15 \pm 2^{\mathrm{ad}}$ & $2.1 \pm 0.6^{\mathrm{a}}$ & $0.9 \pm 0.2^{\mathrm{b}}$ & $4.3 \pm 0.6^{\mathrm{a}}$ & $2.8 \pm 0.4^{\mathrm{b}}$ & $20 \pm 0^{\mathrm{b}}$ \\
\hline $\mathrm{Q}$ & $37 \pm 2^{\text {be }}$ & $11 \pm 0$ & $12 \pm 1^{\mathrm{b}}$ & $16 \pm 1^{\mathrm{ac}}$ & $24 \pm 1$ & $14 \pm 1^{\text {acd }}$ & $1 \pm 0^{\mathrm{a}}$ & $0.1 \pm 0.0^{\mathrm{ac}}$ & $8.7 \pm 1.5$ & $2.6 \pm 0.3$ & $37 \pm 0^{\mathrm{d}}$ \\
\hline $\mathrm{R}$ & $35 \pm 3^{\text {be }}$ & $14 \pm 3$ & $11 \pm 1^{\text {be }}$ & $16 \pm 2^{\mathrm{ac}}$ & $23 \pm 2$ & $14 \pm 1^{\text {acd }}$ & $0.8 \pm 0.3^{\mathrm{a}}$ & $0.09 \pm 0.05^{\mathrm{a}}$ & $6 \pm 0^{\mathrm{a}}$ & $2.4 \pm 0.3$ & $6.1 \pm 0.8^{\mathrm{ae}}$ \\
\hline $\mathrm{S}$ & $39 \pm 3^{b}$ & $8 \pm 0^{\mathrm{b}}$ & $11 \pm 1^{b}$ & $15 \pm 1^{\mathrm{ac}}$ & $23 \pm 2$ & $13 \pm 1^{\text {acd }}$ & $1.7 \pm 0.6^{\mathrm{a}}$ & $0.05 \pm 0.01^{\mathrm{a}}$ & $4.4 \pm 0.5^{\mathrm{a}}$ & $2.1 \pm 0.0$ & $9 \pm 1^{\mathrm{e}}$ \\
\hline $\mathrm{T}$ & $34 \pm 3^{\text {be }}$ & $17 \pm 1^{\mathrm{a}}$ & $11 \pm 0^{\mathrm{b}}$ & $16 \pm 0^{\mathrm{ac}}$ & $24 \pm 1$ & $14 \pm 0^{\mathrm{acd}}$ & $0.7 \pm 0.0^{\mathrm{a}}$ & $0.1 \pm 0.0^{\mathrm{ac}}$ & $7 \pm 0^{\mathrm{a}}$ & $2.7 \pm 0.1$ & $7 \pm 1^{\mathrm{de}}$ \\
\hline \multirow[t]{2}{*}{ Mean } & 28 & 14 & 8 & 14 & 21 & 12 & 2.1 & 0.18 & 7.4 & 2.1 & 13 \\
\hline & PHE & AAA & GLN & ORN & LYS & HIS & TYR & PHP & TRP & $\mathrm{C}-\mathrm{C}$ & Total FAA \\
\hline D & $3.0 \pm 0.1$ & $0.27 \pm 0$ & $00^{\mathrm{ac}} 1.0 \pm$ & 1 & $0^{\mathrm{a}} 7.0$ & & $0.3 \pm 0$ & & $0.7 \pm 0.1^{\mathrm{ac}}$ & & $127^{\mathrm{ac}}$ \\
\hline $\mathrm{E}$ & 4 & $0.26 \pm 0$ & $01^{\text {ac }} 0.8 \pm$ & )$^{\mathrm{a}} 1.9 \pm$ & $2^{\text {a }} 5.3$ & 2.00 .5 & $10.2 \pm$ & $1.3 \pm$ & $0.5 \pm 0.0^{\mathrm{cf}}$ & & $6^{\mathrm{ae}}$ \\
\hline F & $2.7 \pm 0.3$ & $0.25 \pm 0$ & $01^{\mathrm{a}} \quad 0.8 \pm 0$ & )$^{\mathrm{a}} 1.6 \pm$ & $\begin{array}{ll}\mathrm{a} & 6.5 \\
0 & \end{array}$ & $\begin{array}{ll}1.2 & 0.4\end{array}$ & $0.00 .8 \pm$ & $L^{\text {acd }} 1.4 \pm 0.1$ & $0.7 \pm 0.1^{\mathrm{cd}}$ & $0.8 \pm$ & $11^{\mathrm{ae}}$ \\
\hline $\mathrm{H}$ & $2.9 \pm 0.0$ & $0.27 \pm 0$ & $01^{\mathrm{ac}} 0.7 \pm 0$ & $0^{\mathrm{a}} 2.0$ & $3^{\text {a }} 7.7$ & $\begin{array}{lll}0.1 & 0.4\end{array}$ & $0.0 \quad 0.5 \pm$ & $\mathrm{l}^{\text {ac }} 1.3 \pm 0.1$ & $0.6 \pm 0.1^{\mathrm{ce}}$ & \pm 0.0 & 142 \\
\hline I & $2.6 \pm 0.1$ & $0.24 \pm 0$ & $00^{\mathrm{a}} \quad 0.7 \pm 0$ & $0^{\mathrm{a}} 2.8$ & $2^{\mathrm{a}} 4.4$ & $0.30 .4 \pm$ & $0.03 .0 \pm$ & $\mathrm{l}^{\mathrm{e}} \quad 1.3 \pm 0.0$ & $1.1 \pm 0.1^{\text {abde }}$ & $0.7 \pm 0.0$ & $100^{\mathrm{a}}$ \\
\hline $\mathrm{J}$ & $2.4 \pm 0.2^{\mathrm{a}}$ & $0.25 \pm 0$ & $00^{\mathrm{a}} \quad 0.8 \pm 0$ & $.0^{\mathrm{a}} 2.0 \pm$ & $.2^{\mathrm{a}} 5.5$ & $0.90 .5 \pm$ & $0.11 .4 \pm$ & $3^{\text {bdf }} 1.3 \pm 0.0$ & $1.1 \pm 0.1^{\mathrm{abd}}$ & $0.7 \pm 0.0$ & $105^{\mathrm{ae}}$ \\
\hline $\mathrm{K}$ & $2.9 \pm 0.2$ & $0.26 \pm 0$ & $00^{\mathrm{ac}} 0.8 \pm 0$ & $1^{\mathrm{a}} 1.3 \pm$ & $2^{\mathrm{a}} 7.4 \pm$ & $0.90 .5 \pm$ & $0.10 .9 \pm 0.3$ & $3^{\text {acd }} 1.3 \pm 0.0$ & $0.4 \pm 0.0^{\mathrm{c}}$ & $0.9 \pm 0.1$ & $133^{\text {ace }}$ \\
\hline $\mathrm{L}$ & $2.8 \pm 0.0$ & $0.29 \pm 0$ & $01^{\mathrm{ac}} 0.8 \pm 0$ & $0^{\mathrm{a}} 1.3 \pm$ & $0.3^{\mathrm{a}} 7.0 \pm$ & $0.20 .5 \pm$ & $0.10 .3 \pm 0.0$ & )$^{\text {ac }} 1.3 \pm 0.0$ & $0.5 \pm 0.1^{\mathrm{cf}}$ & $0.8 \pm 0.0$ & $133^{\mathrm{ac}}$ \\
\hline M & $2.5 \pm 0.1$ & $0.25 \pm 0$ & $00^{\mathrm{a}} \quad 0.8 \pm 0$ & $.^{\mathrm{a}} 1.4 \pm$ & $.1^{\mathrm{a}} 6.2 \pm$ & $0.50 .5 \pm$ & $0.0 \quad 0.3 \pm 0.1$ & ${ }^{\text {ac }} 1.3 \pm 0.0$ & $1.3 \pm 0.1^{\mathrm{b}}$ & $0.7 \pm 0.0$ & $109^{\mathrm{ae}}$ \\
\hline $\mathrm{N}$ & $2.8 \pm 0.1$ & $0.24 \pm 0$ & $00^{\mathrm{a}} \quad 1 \pm 0$ & $5.4 \pm$ & $1.6^{b} 6.3 \pm$ & $0.50 .4 \pm$ & $0.0 \quad 2.1 \pm 0.1$ & $l^{\text {eg }} \quad 1.3 \pm 0.1$ & $1.2 \pm 0.1^{\mathrm{ab}}$ & $0.7 \pm 0.0$ & $194^{\text {bd }}$ \\
\hline $\mathrm{O}$ & $3.2 \pm 0.2$ & $0.42 \pm 0$ & $01^{\mathrm{b}} 0.8 \pm 0$ & $0^{\mathrm{a}} 1.5 \pm$ & $.4^{\mathrm{a}} 8.1 \pm$ & $0.80 .3 \pm$ & $0.0 \quad 0.5 \pm 0.0$ & )$^{\text {acf }} 1.4 \pm 0.1$ & $0.6 \pm 0.1^{\mathrm{ce}}$ & $0.9 \pm 0.1$ & $164^{\text {ade }}$ \\
\hline $\mathrm{P}$ & $3.4 \pm 0.3^{\mathrm{b}}$ & $0.26 \pm 0$ & $00^{\mathrm{a}} \quad 1.3 \pm 0$ & $2^{\mathrm{b}} 1.9 \pm$ & $.2^{\mathrm{a}} 8.0 \pm$ & $1.60 .4 \pm$ & $0.10 .5 \pm 0.1$ & $\mathrm{~L}^{\text {acf }} 1.4 \pm 0.1$ & $1.0 \pm 0.1^{\text {abdef }}$ & $0.9 \pm 0.1$ & $165^{\text {ade }}$ \\
\hline $\mathrm{Q}$ & $3.1 \pm 0.1$ & $0.36 \pm 0$ & $06^{\mathrm{bc}} 0.8 \pm 0$ & $\begin{array}{ll}0^{\mathrm{a}} & 1.6 \pm\end{array}$ & $.0^{\mathrm{a}} 7.2 \pm$ & $0.80 .4 \pm$ & $0.0 \quad 1.4 \pm 0.4$ & $7^{\text {bdg }} 1.5 \pm 0.1$ & $0.7 \pm 0.1^{\mathrm{cd}}$ & $0.8 \pm 0.1$ & $181^{\text {cd }}$ \\
\hline $\begin{array}{l}\mathrm{Q} \\
\mathrm{R}\end{array}$ & $3.0 \pm 0.1$ & $0.29 \pm 0$ & $03^{\mathrm{ac}} 0.7 \pm 0$ & 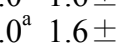 & $.3^{\mathrm{a}} 7.3 \pm$ & $1.30 .4 \pm$ & $0.0 \quad 1.0 \pm 0.2$ & $2^{\text {acd }} 1.4 \pm 0.1$ & $0.6 \pm 0.0^{\mathrm{ce}}$ & $0.8 \pm 0.0$ & 146 \\
\hline $\mathrm{S}$ & $3.0 \pm 0.1$ & $0.26 \pm 0$ & $01^{\mathrm{a}} \quad 0.8 \pm 0$ & $.0^{\mathrm{a}} 1.2 \pm$ & $0.2^{\mathrm{a}} 7.8 \pm$ & $0.40 .4 \pm$ & $0.0 \quad 1.1 \pm 0.2$ & $2^{\mathrm{cd}} \quad 1.3 \pm 0.0$ & $0.8 \pm 0.1^{\mathrm{ac}}$ & $0.8 \pm 0.0$ & 144 \\
\hline $\mathrm{T}$ & $3.1 \pm 0.1$ & $0.31 \pm 0$ & $01^{\mathrm{ac}} 0.7 \pm 0$ & $.0^{\mathrm{a}} 1.7 \pm$ & $.1^{\mathrm{a}} 8.2 \pm$ & $0.20 .5 \pm$ & $0.1 \quad 1.0 \pm 0.1$ & acd $1.5 \pm 0.0$ & $0.8 \pm 0.2^{\mathrm{ac}}$ & $0.9 \pm 0.0$ & 152 \\
\hline Mean & 2.9 & 0.28 & 0.8 & 1. & & $\begin{array}{ll}9 & 0.4\end{array}$ & 1.0 & 1.4 & 0.8 & 0.8 & 139 \\
\hline
\end{tabular}

Data are presented as mean \pm SE for 3 replicates. The values with different superscript letters differ with each other at the significant level of $\mathrm{p}<0.05$. The mean values with no superscript letter mean that this amino acid in this brand is nearly the same as that in all other brands.

$\mathrm{C}_{18: 2(\mathrm{n}-6)}$ was especially the highest in all fatty acids. Products from S. China had the highest free fatty acid contents $(830 \mathrm{mg} / 100 \mathrm{~g}$ fresh sample) and were followed by Hong Kong (706). Products from N. China and Taiwan were comparable in the free fatty acids content at 636 and $639 \mathrm{mg} / 100 \mathrm{~g}$ fresh sample, respectively (Tables 4 and 5). Especially, $\mathrm{C}_{18: 2(\mathrm{n}-6)}, \mathrm{C}_{18: 3}$ and $\mathrm{C}_{18: 1(\mathrm{n}-9) \text {, }}$ which were found in relatively higher amount, were not only the essential fatty acids, but also the precursors for the desired flavor of sufu. Chung (12) found numerous special esters in volatile components of commercial sufus. Those esters were converted from the above three fatty acids esterified with alcohol and might contribute sour, meaty, coconut-like and sweaty flavor to sufu odor $(12,15)$. It is believed that, if more of these desired fatty acids are released during fermentation, the taste and odor of sufu will be much more attractive.

To date, there are no uniform criteria existing for determining whether the quality and maturation of sufu is good or not in the sufu industry for its production. Although the basic production procedures of sufu are the same, the production method, raw materials, production seasons and the fermentation period usually vary from one manufacture to another. Thus, any deviation in the process of production may result in very different products. It is hoped that the information found in this investigation is able to provide some important quality reference ranges for product developers and manufacturers to optimize and produce their fermented soybean curds. 
Table 4. Retention times, elution order, names, abbreviations and ion fragments of the 30 free fatty acids

\begin{tabular}{|c|c|c|c|c|}
\hline Rt (min) & Elution order & Chemical name & Abbreviation & Mass fragment ions \\
\hline 1.42 & 1 & Butanoic acid & $\mathrm{C}_{4: 0}$ & 73,88 \\
\hline 1.65 & 2 & n-Valeric acid & $\mathrm{C}_{5: 0}$ (I.S.) & $73,87,93$ \\
\hline 2.07 & 3 & Hexanoic acid & $\mathrm{C}_{6: 0}$ & $73,87,80$ \\
\hline 2.83 & 4 & Hexanoic acid & $\mathrm{C}_{7: 0}$ & 87,101 \\
\hline 3.85 & 5 & Octanoic acid & $\mathrm{C}_{8: 0}$ & 73,101 \\
\hline 4.64 & 6 & Nonanoic acid & $\mathrm{C}_{9: 0}$ & 73,115 \\
\hline 5.27 & 7 & Decanoic acid & $\mathrm{C}_{10: 0}$ & $73,129,143$ \\
\hline 5.78 & 8 & Undecanoic acid & $\mathrm{C}_{11: 0}$ & $73,129,85$ \\
\hline 6.22 & 9 & Dodecanoic acid & $\mathrm{C}_{12: 0}$ & $73,129,157$ \\
\hline 6.60 & 10 & Tridecanoic acid & $\mathrm{C}_{13: 0}$ & $73,129,171$ \\
\hline 6.95 & 11 & Tetradecanoic acid & $\mathrm{C}_{14: 0}$ & $73,129,185$ \\
\hline 7.27 & 12 & Pentadecanoic acid & $\mathrm{C}_{15: 0}$ & $73,129,199$ \\
\hline 7.53 & 13 & cis-9-Hexadecenoic acid & $\mathrm{C}_{16: 1}$ & $83,97,70$ \\
\hline 7.58 & 14 & Hexadecanoic acid & $\mathrm{C}_{16: 0}$ & $73,129,256$ \\
\hline 7.86 & 15 & Heptadecanoic acid & $\mathrm{C}_{17: 0}$ (I.S.) & $73,129,270$ \\
\hline 8.08 & 16 & Linoleic acid & $\mathrm{C}_{18: 2(\mathrm{n}-9)}$ & $81,95,109$ \\
\hline 8.09 & 17 & Oleic acid & $\mathrm{C}_{18: 1(\mathrm{n}-9)}$ & $83,97,111$ \\
\hline 8.10 & 18 & Linolenic acid & $\mathrm{C}_{18: 3}$ & $79,93,108$ \\
\hline 8.13 & 19 & Octadecanoic acid & $\mathrm{C}_{18: 0}$ & 73,129 \\
\hline 8.39 & 20 & Nonadecanoic acid & $\mathrm{C}_{19: 0}$ & $73,129,298$ \\
\hline 8.53 & 21 & Arachidonic acid & $\mathrm{C}_{20: 4}$ & $79,91,105$ \\
\hline 8.55 & 22 & cis-5,8,11,14,17-Eicosapentaenoic acid & $\mathrm{C}_{20: 5}$ & $79,91,105$ \\
\hline 8.64 & 23 & Arachidic acid & $\mathrm{C}_{20: 0}$ & $73,129,312$ \\
\hline 8.88 & 24 & Heneicosanoic acid & $\mathrm{C}_{21: 0}$ & $73,326,129$ \\
\hline 9.02 & 25 & cis-4,7,10,13,16,19-Docosahexaenoic acid & $\mathrm{C}_{22: 6}$ & $79,91,105$ \\
\hline 9.09 & 26 & Erucic acid & $\mathrm{C}_{22: 1}$ & $83,97,111$ \\
\hline 9.14 & 27 & Docosanoic acid & $\mathrm{C}_{22: 0}$ & $73,340,129$ \\
\hline 9.40 & 28 & Tricosanoic acid & $\mathrm{C}_{23: 0}$ & $73,129,354$ \\
\hline 9.65 & 29 & Nervonic acid & $\mathrm{C}_{24: 1}$ & $83,97,111$ \\
\hline 9.70 & 30 & Tetracosanoic acid & $\mathrm{C}_{24: 0}$ & $73,368,129$ \\
\hline
\end{tabular}

Table 5. Free fatty acid contents of different sufu brands

\begin{tabular}{|c|c|c|c|c|c|c|c|c|c|c|c|}
\hline \multirow[t]{2}{*}{ Brand } & \multicolumn{11}{|c|}{$\begin{array}{l}\text { Free fatty acid concentration ( } \mathrm{mg} / 100 \mathrm{~g} \text { fresh weight of sufu) } \\
\text { (mean } \pm \text { SE for } 3 \text { replicates) }\end{array}$} \\
\hline & $\mathrm{C}_{4: 0}$ & $\mathrm{C}_{8: 0}$ & $\mathrm{C}_{14: 0}$ & $\mathrm{C}_{16: 1}$ & $\mathrm{C}_{16: 0}$ & $\mathrm{C}_{18: 2(\mathrm{n}-6)}$ & $\mathrm{C}_{18: 1(\mathrm{n}-9)}$ & $\mathrm{C}_{18: 3}$ & $\mathrm{C}_{18: 0}$ & $\mathrm{C}_{20: 0}$ & Total FFA \\
\hline D & $0.7 \pm 0.1^{\mathrm{a}}$ & $0.9 \pm 0.1$ & $0.7 \pm 0.0$ & $0.7 \pm 0.0$ & $70 \pm 3^{\mathrm{ac}}$ & $489 \pm 10^{\text {acef }}$ & $78 \pm 6^{\mathrm{ac}}$ & $6 \pm 0^{\mathrm{a}}$ & $35 \pm 5^{\text {ade }}$ & $1.5 \pm 0.2$ & $683^{\mathrm{ac}}$ \\
\hline $\mathrm{E}$ & $0.9 \pm 0.2^{\mathrm{a}}$ & $0.8 \pm 0.0^{\mathrm{a}}$ & $0.6 \pm 0.1$ & $0.6 \pm 0.1$ & $51 \pm 0^{\mathrm{a}}$ & $172 \pm 3^{\mathrm{b}}$ & $52 \pm 8^{\mathrm{a}}$ & $5 \pm 0^{\mathrm{a}}$ & $16 \pm 0^{\mathrm{ce}}$ & $1.0 \pm 0.1^{\mathrm{a}}$ & $299^{c}$ \\
\hline $\mathrm{F}$ & $0.6 \pm 0.1^{\mathrm{a}}$ & $0.8 \pm 0.1$ & $0.8 \pm 0.1$ & $0.7 \pm 0.0$ & $96 \pm 0$ & $391 \pm 6^{\mathrm{cfi}}$ & $133 \pm 9$ & $8 \pm 0$ & $41 \pm 6$ & $1.8 \pm 0.0$ & $674^{\text {ad }}$ \\
\hline $\mathrm{H}$ & $2.3 \pm 0.2^{\mathrm{ac}}$ & $0.9 \pm 0.1$ & $1.1 \pm 0.2$ & $0.9 \pm 0.1$ & $165 \pm 30^{c}$ & $558 \pm 13^{\text {cdef }}$ & $138 \pm 23$ & $11 \pm 2$ & $56 \pm 5^{\mathrm{bd}}$ & $1.8 \pm 0.1$ & $936^{\mathrm{ab}}$ \\
\hline I & $1.5 \pm 0.1^{\mathrm{a}}$ & $0.9 \pm 0.0$ & $1.2 \pm 0.3$ & $0.9 \pm 0.2$ & $88 \pm 6^{\mathrm{ac}}$ & $312 \pm 12^{\text {cfgh }}$ & $113 \pm 7$ & $7 \pm 1^{\mathrm{ac}}$ & $34 \pm 3^{\text {ade }}$ & $1.6 \pm 0.1$ & $560^{\mathrm{ac}}$ \\
\hline $\mathrm{J}$ & $1.7 \pm 0.4^{\mathrm{a}}$ & $0.8 \pm 0.0^{\mathrm{a}}$ & $0.6 \pm 0.1$ & $0.8 \pm 0.1$ & $57 \pm 4^{\mathrm{a}}$ & $690 \pm 13^{\mathrm{di}}$ & $126 \pm 28$ & $9 \pm 1$ & $25 \pm 4^{\mathrm{ef}}$ & $1.9 \pm 0.4$ & $913^{\mathrm{ab}}$ \\
\hline $\mathrm{K}$ & $1.3 \pm 0.1^{\mathrm{a}}$ & $0.9 \pm 0.1$ & $0.8 \pm 0.0$ & $0.7 \pm 0.1$ & $93 \pm 0$ & $243 \pm 33^{\text {bghj }}$ & $83 \pm 11^{\text {ac }}$ & $6 \pm 0^{\mathrm{a}}$ & $54 \pm 0^{\text {bd }}$ & $2.0 \pm 0.4$ & $484^{\text {cd }}$ \\
\hline $\mathrm{L}$ & $4.8 \pm 0.3^{\mathrm{a}}$ & $1.3 \pm 0.0^{\mathrm{b}}$ & $1.1 \pm 0.1$ & $0.8 \pm 0.0$ & $190 \pm 18^{b}$ & $726 \pm 19^{\mathrm{di}}$ & $206 \pm 12^{\mathrm{cd}}$ & $15 \pm 2^{\mathrm{b}}$ & $60 \pm 7^{\mathrm{bd}}$ & $2.4 \pm 0.2^{\mathrm{b}}$ & $1203^{b}$ \\
\hline M & $2.3 \pm 0.3^{\mathrm{ac}}$ & $0.9 \pm 0.0^{\mathrm{a}}$ & $1.1 \pm 0.1$ & $0.8 \pm 0.0$ & $162 \pm 45^{c}$ & $568 \pm 65^{\text {cdefi }}$ & $194 \pm 38$ & $14 \pm 2^{\mathrm{bd}}$ & $52 \pm 9^{\text {bd }}$ & $1.7 \pm 0.1$ & $997^{\mathrm{ab}}$ \\
\hline $\mathrm{N}$ & $1.7 \pm 0.1^{\mathrm{ac}}$ & $0.9 \pm 0.1$ & $0.9 \pm 0.1$ & $0.8 \pm 0.1$ & $101 \pm 9$ & $357 \pm 45^{\text {abghj }}$ & $193 \pm 48$ & $8 \pm 2^{\text {acd }}$ & $53 \pm 6^{\text {bd }}$ & $1.7 \pm 0.2$ & $718^{\mathrm{ad}}$ \\
\hline $\mathrm{O}$ & $4.2 \pm 0.2^{b c}$ & $0.9 \pm 0.1$ & $1.1 \pm 0.2$ & $0.8 \pm 0.0$ & $117 \pm 12$ & $632 \pm 13^{\text {edi }}$ & $155 \pm 18$ & $6 \pm 0^{\mathrm{a}}$ & $51 \pm 6^{\mathrm{bdf}}$ & $1.9 \pm 0.3$ & $968^{\mathrm{ab}}$ \\
\hline $\mathrm{P}$ & $0.8 \pm 0.1^{\mathrm{a}}$ & $0.9 \pm 0.0$ & $0.8 \pm 0.0$ & $0.7 \pm 0.1$ & $99 \pm 28$ & $395 \pm 46^{\mathrm{cfg}}$ & $111 \pm 7$ & $9 \pm 2$ & $32 \pm 0^{\text {ade }}$ & $1.4 \pm 0.1$ & $649^{\mathrm{ac}}$ \\
\hline $\mathrm{Q}$ & $1.8 \pm 0.4^{\mathrm{a}}$ & $0.8 \pm 0.0^{\mathrm{a}}$ & $0.7 \pm 0.0$ & $0.7 \pm 0.0$ & $84 \pm 18^{\mathrm{ac}}$ & $278 \pm 54^{\mathrm{bghj}}$ & $90 \pm 22$ & $8 \pm 1^{\text {acd }}$ & $31 \pm 1^{\text {ade }}$ & $1.4 \pm 0.1$ & $497^{\mathrm{cd}}$ \\
\hline $\mathrm{R}$ & $1.6 \pm 0.3^{\mathrm{a}}$ & $1.1 \pm 0.3$ & $0.8 \pm 0.2$ & $0.7 \pm 0.1$ & $62 \pm 6^{\mathrm{a}}$ & $285 \pm 11^{\text {bghj }}$ & $76 \pm 12^{\mathrm{ac}}$ & $6 \pm 0^{\mathrm{a}}$ & $32 \pm 3^{\text {ade }}$ & $1.5 \pm 0.3$ & $467^{\mathrm{cd}}$ \\
\hline S & $2.1 \pm 0.4^{\mathrm{a}}$ & $0.9 \pm 0.0^{\mathrm{a}}$ & $1.1 \pm 0.2$ & $1.0 \pm 0.2$ & $134 \pm 24$ & $716 \pm 6^{\mathrm{d}}$ & $233 \pm 6^{\mathrm{bd}}$ & $13 \pm 2^{\mathrm{bc}}$ & $65 \pm 1^{b}$ & $2.0 \pm 2.0$ & $1168^{\mathrm{b}}$ \\
\hline $\mathrm{T}$ & $5.1 \pm 1.1^{\mathrm{b}}$ & $0.9 \pm 0.1$ & $0.8 \pm 0.1$ & $0.7 \pm 0.1$ & $107 \pm 2$ & $425 \pm 26^{\mathrm{fj}}$ & $149 \pm 0$ & $9 \pm 1$ & $34 \pm 4^{\text {ade }}$ & $1.4 \pm 0.1$ & $732^{\mathrm{ad}}$ \\
\hline Mean & 2.1 & 0.9 & 0.9 & 0.8 & 105 & 452 & 133 & 9 & 42 & 1.7 & 747 \\
\hline
\end{tabular}

Data are presented as mean \pm SE for 3 replicates. The values with different superscript letters differ with each other at the significant level of $\mathrm{p}<0.05$. The mean values with no superscript letter mean that this fatty acid in this brand is nearly the same as that in all other brands.

\section{ABBREVIATIONS}

TPA, texture profile analysis; SD, standard; SE, standard error; FAA, free amino acid; FFA, free fatty acid;
SPE, solid phase extraction; GC/MS, gas chromatography-mass spectrometry; MSD, mass spectrometry detector. 


\section{REFERENCES}

1. Reddy NR, Pierson MD, Salunkhe DK. 1986. Legumebased fermented foods. CRC Press, Boca Raton, FL, USA. p 254.

2. Steinkraus KH. 1996. Chinese sufu. In Handbook of Indigenous Fermented Foods. Marcel Dekker, Inc., New York, NY, USA. p 69-83.

3. Akın N, Aydemir S, Koçak C, Yıldız M. 2003. Changes of free fatty acid contents and sensory properties of white pickled cheese during ripening. Food Chem 80: 77-83.

4. AOAC. 1996. Official methods of analysis. 17th ed. Association of Official Analytical Chemists, Washington, DC, USA.

5. Lim BT, Deman JM, Deman L, Buzzell RI. 1990. Yield and quality of tofu as coagulant. J Food Sci 55: 1088-1092.

6. AOAC. 1995. Official methods of analysis. 16th ed. Association of Official Analytical Chemists, Washington, DC, USA.

7. Nozal MJ, Bernal JL, Toribio ML, Diego JC, Ruiz A. 2004. Rapid and sensitive method for determining free amino acids in honey by gas chromatography with flame ionization or mass spectrometric detection. J Chromatogr A 1047: 137-146.

8. Lee J, Harnly JM. 2005. Free amino acid and cysteine sulfoxide composition of 11 garlic (Allium sativum L.) cultivars by gas chromatography with flame ionization and mass selective detection. J Agric Food Chem 53: 91009104.

9. Deeth HC, Fitz-Gerald CH, Snow AJ. 1983. A gas chromatographic method for the quantitative determination of free fatty acids in milk and milk products. New Zeal J Dairy Sci 18: 13-20.

10. Bourne MC. 2002. Texture profile analysis. In Food Texture and Viscosity: Concept and Measurement. 2nd ed. Academic Press, New York, NY, USA. p 182-186.

11. Gunasekaran S, Mehmet MA. 1957. Cheese texture. In Cheese Rheology and Texture. CRC Press LLC, Boca Raton, FL, USA. p 299-325.

12. Chung HY. 1999. Volatile components in fermented soybean (Glycine max) curds. J Agric Food Chem 47: 26902696.

13. Chung HY. 2000. Volatile flavour components in red fermented soybean (Glycine max) curds. J Agric Food Chem 48: 1803-1809.

14. Benito MJ, Rodríguez M, Martín A, Aranda E, Cordoba JJ. 2004. Effect of the fungal protease EPg222 on the sensory characteristics of dry fermented sausage "salchichón" ripened with commercial starter cultures. Meat Sci 67: 497-505.

15. Chung HY, Fung PK, Kim JS. 2005. Aroma impact components in commercial plain sufu. J Agric Food Chem 53: 1684-1691.

(Received February 22, 2011; Accepted March 8, 2011) 